\title{
Electrochemical, surface analytical and quantum chemical studies on Schiff bases of 4-amino-4H-1, 2, 4-triazole-3,5-dimethanol (ATD) in corrosion protection of aluminium in $1 \mathrm{~N} \mathrm{HNO}_{3}$
}

\author{
SAM JOHN, K MOHAMMAD ALI and ABRAHAM JOSEPH* \\ Department of Chemistry, University of Calicut, Calicut 673 635, India
}

MS received 30 November 2010; revised 14 February 2011

\begin{abstract}
The present study describes the inhibition of aluminium in $1 \mathrm{~N} \mathrm{HNO}_{3}$ with different concentrations of 1,2,4-triazole precursors ATD, BATD and DBATD using gravimetric method, potentiodynamic polarization studies (Tafel), electrochemical impedance spectroscopy (EIS), adsorption studies, surface morphological studies and quantum chemical calculations at $298 \mathrm{~K}$. Polarization studies clearly showed that ATD, BATD and DBATD act as mixed type inhibitors. As the electron density around the inhibitor molecule increases due to substitution, the inhibition efficiency also increases correspondingly. Quantum chemical approach was used to calculate some electronic properties of the molecule to ascertain the correlation between inhibitive effect and molecular structure of the inhibitor. The corrosion inhibition efficiencies of these molecules and the global chemical reactivity relate to some parameters, such as $E_{\text {HOMO }}, E_{\text {LUMO }}$, gap energy $(\Delta E)$, electronegativity $(\chi)$, global hardness $(\eta)$ and the fraction of electrons transferred from the inhibitor molecule to the metallic atom $(\Delta N)$. In addition, the local reactivity has been analysed through the Fukui function and condensed softness indices. Both the experimental and theoretical studies agree well in this regard and confirm that DBATD is a better inhibitor than BATD and ATD. The adsorption behaviours of molecules on the copper surface have been studied using molecular dynamics method and density functional theory. The order of inhibitory action is DBATD > BATD > ATD.
\end{abstract}

Keywords. Aluminium; acid solutions; EIS; polarization.

\section{Introduction}

Aluminium is an extremely valuable material due to its light weight, high strength, recyclability, corrosion resistance, durability, ductility, formability, and conductivity. Aluminium and its alloys find extensive applications with various industries in different capacities. Aluminium and its alloys, however, are reactive materials and are prone to corrosion. Corrosion of aluminium and its alloys has been a subject of numerous studies due to their high technological value and wide range of industrial applications in aerospace and house hold industries. Though aluminium facilitates the formation of a compact, adherent passive oxide film for its corrosive immunity in various environments, the surface film is amphoteric and dissolves substantially when the metal is exposed to high concentrations of acids or bases (Pourbaix 1966; Khaled and Al-Qahtani 2009). The solubility of the oxide film increases above or below $\mathrm{pH}$ 4-9 range and the metal exhibits uniform attack. The use of corrosion inhibitors is inevitable under these circumstances. Most of the efficient acid corrosion inhibitors contain hetero atoms such as $\mathrm{N}, \mathrm{O}$, $\mathrm{S}$ and multiple bonds in their molecules which activate the process of adsorption. This adsorption process gets activated

\footnotetext{
*Author for correspondence (drabrahamj@gmail.com)
}

as the electron donating efficiency of the inhibitor molecule increases. Most of the hetero atoms in organic molecules bear non-bonding electro pairs in the valence shell and hence possess excellent inhibitory action. The adsorption of molecules on the metal surface is also influenced by their electronic structure, steric factors, aromaticity and $p$-orbital character of donating electrons (Dessai et al 1986; Ajmal et al 1994; Abd El-Maksoud 2002).

The aim of the present study is to examine the influence of some selected triazole derivatives on the corrosion inhibition of aluminium in $1 \mathrm{~N} \mathrm{HNO}_{3}$ solutions using gravimetric, electrochemical techniques, polarization and electrochemical impedance spectroscopy as well as explicit simulations using molecular dynamics and quantum chemical calculations.

\section{Experimental}

\subsection{Inhibitor}

The inhibitor molecule 1, 2, 4-triazole precursor, 4-amino4H-1, 2, 4-triazole-3, 5-dimethanol (ATD) was synthesized by the condensation of glycolic acid with hydrazine hydrate. Hydrazine monohydrate (E. Merck) (3.75 g, $0.75 \mathrm{~mol})$ was added drop wise at $0^{\circ} \mathrm{C}$ to $70 \%$ aqueous glycolic acid 
(E. Merck) $(54.3 \mathrm{~g}, 0.50 \mathrm{~mol})$. The resulting solution was heated at $120^{\circ} \mathrm{C}$ for $6 \mathrm{~h}$. Then the reflux condenser was replaced with a downward condenser and reaction mixture was heated at $160^{\circ} \mathrm{C}$ for a further $18 \mathrm{~h}$ allowing excess hydrazine and water to distil off. The yellowish crystalline solid formed after cooling was recrystallized from water ATD (Klingele et al 2005). (4-(Benzylideneamino)4H-1, 2, 4-triazole-3,5-diyl) dimethanol (BATD) and (4-(4(dimethylamino) benzylideneamino))-4H-1,2,4-triazole-3,5diyl) dimethanol (DBATD) (E. Merck) (1:1 in molar ratio) were prepared by the condensation of ATD with benzaldehyde (E. Merck) (1:1 in molar ratio) and dimethylaminobenzaldehyde (E. Merck) (1:1 in molar ratio), respectively using alcohol as the solvent. The structure of the inhibitor molecules are shown in figures $1 \mathrm{a}-\mathrm{c}$.

\subsection{Medium}

The medium for the study was made from reagent grade $\mathrm{HNO}_{3}$ (E. Merck) and doubly distilled water. All tests were

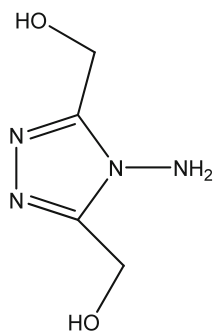

4-amino-4H-1, 2, 4-triazole -3,5-dimethanol(ATD) (a)

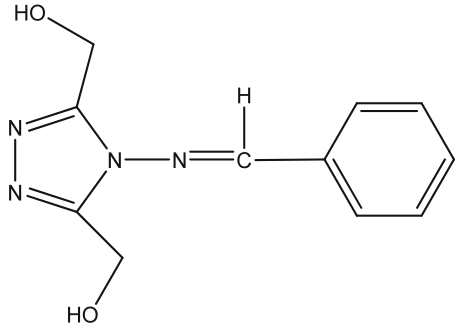

(4-(benzylideneamino)-4H-1, 2, 4-triazole-3, 5-diyl)dimethanol(BATD) (b)

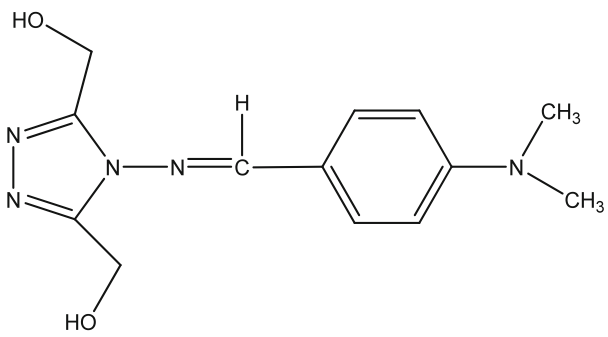

(4-(4-(dimethylamino)benzylideneamino))-4H-1,2,4-triazole-3,5-diyl)dimethanol (DBATD) (c)

Figure 1. Molecular structure of studied triazole derivatives: (a) 4-amino-4H-1, 2, 4-triazole-3,5-dimethanol(ATD), (b) (4-(benzylideneamino)-4H-1, 2, 4-triazole-3, 5-diyl) dimethanol (BATD) and (c) (4-(4-(dimethylamino)benzylideneamino))-4H-1,2,4triazole-3,5-diyl) dimethanol (DBATD). performed in aerated medium at room temperature and atmospheric pressure.

\subsection{Materials}

The working electrode was of $100 \%$ pure aluminium as determined by EDX spectra. The metal specimens used in weight loss studies are cut into $4.8 \times 1.9 \mathrm{~cm}^{2}$ coupons. For electrochemical studies, same type of coupons was used but only $1 \mathrm{~cm}^{2}$ area was exposed during each measurements. Before measurements the samples were polished using different grades of emery paper followed by washing with ethanol and acetone.

\subsection{Weight loss measurements}

The weight loss experiments were carried out under total immersion conditions in test solution maintained at $300 \mathrm{~K}$. Metal specimens were cleaned according to ASTM standard G-1-72 procedure (Ailor 1971; Talati and Modi 1975; Bag et al 1993; McCafferty et al 1999). The experiments were carried out in a beaker containing $250 \mathrm{ml}$ solution. After the exposure period the specimens were removed, washed initially under running tap water, to remove the loosely adhering corrosion products and finally cleaned with $70 \% \mathrm{HNO}_{3}$ for 3 min followed by acetone. Experiments were conducted at the same temperature with different inhibitor concentrations to find out the optimum inhibitor concentration that shows maximum inhibitive efficiency. From the weight loss in each experiment the corrosion rate was calculated in milli meter/year (mm/yr). In each case, duplicate experiments were conducted which showed that the results obtained were within $\pm 1 \%$ of the first. Whenever variations were very large, the data were confirmed by a third test. The percentage inhibitive efficiency was calculated using the relation

$$
\% \mathrm{IE}=\frac{W_{0}-W}{W_{0}} \times 100,
$$

where $W_{0}$ and $W$ are the weight losses in the uninhibited and inhibited solutions, respectively.

\subsection{Electrochemical measurements}

Electrochemical tests were carried out in a three-electrode corrosion cell with platinum sheet $\left(1 \mathrm{~cm}^{2}\right.$ surface area) as auxiliary electrode and saturated calomel electrode (SCE) as the reference electrode. The working electrode was first immersed in the test solution and after attaining a steady state open circuit potential (OCP), electrochemical measurements were carried out with a Gill AC computer controlled electrochemical workstation (ACM, UK, model no: 1475). Electrochemical impedance spectroscopy (EIS) measurements were carried out with an amplitude of $10 \mathrm{mV}$ (RMS) AC sine wave in a frequency range of $10 \mathrm{kHz}$ to $1 \mathrm{~Hz}$. The potentiodynamic 
polarization curves were obtained in the potential range of $-250 \mathrm{mV}$ to $+250 \mathrm{mV}$ with a sweep rate of $16 \mathrm{mV} / \mathrm{s}$.

\subsection{Computational study}

Complete geometry optimization of the metal specimen are performed using the density functional theory (DFT) with Beck's three parameter exchange functional along with LeeYang-Parr non-local correlation functional (B3LYP) with 6-31G* basis set implemented in Gaussian 03 program package (Frisch et al 2003; Ebenso et al 2010). Frontier molecular orbitals (HOMO and LUMO) may be used to interpret the adsorption characteristics of inhibitor molecules on the metal surface.

The local reactivity of the inhibitor molecules was analysed through an evaluation of the Fukui indices (Arslan et al 2009). Fukui indices are a measurement of the chemical reactivity, as well as an indicator of the reactive regions and the nucleophilic and electrophilic behaviours of the molecule. The regions of a molecule where the Fukui function is large are chemically softer than the regions where the Fukui function is small, and by invoking HSAB principle in a local sense, one may establish the behaviour of different sites with respect to hard or soft reagents. The Fukui function $f(\vec{r})$ is defined as the derivative of the electronic density, $\rho(\vec{r})$, with respect to the number of electrons, $N$, at a constant external potential, $\sigma(\vec{r})$

$$
f(\vec{r})=\left(\frac{\partial \rho(\vec{r})}{\partial N}\right)_{\sigma(\vec{r})} .
$$

If the effects of relaxation associated with the addition or removal of electronic charges are not considered, then

$$
\begin{aligned}
& \rho^{+}(\vec{r}) \approx \rho_{\text {LUMO }}(\vec{r}), \\
& \rho^{-}(\vec{r}) \approx \rho_{\mathrm{HOMO}}(\vec{r}),
\end{aligned}
$$

where $\rho_{\text {LUMO }}(\vec{r})$ is the density of the lowest unoccupied molecular orbital and $\rho_{\mathrm{HOMO}}(\vec{r})$ the density of the highest occupied molecular orbital (Yang and Mortier 1986; Gece 2008; Solmaz et al 2008). The condensed Fukui functions are found by taking the finite difference approximations from Mulliken population analysis of atoms in triazole derivatives, depending on the direction of the electron transfer

$$
\begin{aligned}
& f_{k}^{+}=q_{k}(N+1)-q_{k}(N), \\
& f_{k}^{-}=q_{k}(N)-q_{k}(N-1),
\end{aligned}
$$

where $q_{k}$ is the gross charge of atom, $k$, in the molecule i.e. the electron density at a point $r$ in space around the molecule. $N$ corresponds to the number of electrons in the neutral molecule, $N+1$ corresponds to an anion, with an electron added to the LUMO of the neutral molecule and
$N-1$ represents the cation with an electron removed from HOMO of the neutral molecule. All calculations are done at the ground-state geometry. These functions can be condensed to the nuclei by using an atomic charge partitioning scheme, such as Mulliken population analysis in (3)-(6). An easy graphical display technique was also used based on the Fukui functions. Instead of calculating the molecular orbitals for the neutral, cation, and anion, we can just add or subtract electrons from the molecular orbitals of the neutral molecule. Though this procedure is not as good as the first method, it does give a quick graphical display of the susceptibility to different kinds of attack.

The molecular dynamics (MD) simulation was performed using Material Studio 4.3 software from Accelrys Inc. $\mathrm{Al}(110)$ plane was chosen for the simulation study. The interaction energy, $E_{\text {interaction }}$, between the aluminium surface and the inhibitor molecule was calculated as

$$
E_{\text {interaction }}=E_{\text {total }}-\left(E_{\text {surface }}+E_{\text {inhibitor }}\right),
$$

where $E_{\text {total }}$ is the total energy of Al-metal and inhibitor molecules. $E_{\text {surface }}$ and $E_{\text {inhibitor }}$ are the energy of aluminium and inhibitor molecules, respectively. The binding energy of the inhibitor molecule is the negative value of the interaction energy

$$
E_{\text {binding }}=-E_{\text {interaction }} \text {. }
$$

\subsection{Scanning electron microscopy (SEM)}

The study of the surface morphology of the metal specimen in both absence and presence of inhibitor molecules were carried out by using a Digital Microscope Imaging Scanning Electron Microscope model SU6600 (Serial No: HI$2102-0003$ ) with an accelerating voltage of $20 \mathrm{kV}$, at a scan speed $=$ slow 5 and calibration scan speed $=25$. Samples were attached on the top of an aluminium stopper by means of a carbon conductive adhesive tape. All micrographs of the specimen were taken at a magnification of $500 \times$.

\section{Results and discussion}

\subsection{Weight loss measurements}

Weight loss studies were performed at various time intervals in the absence and presence of different concentrations (10-200 ppm) of ATD, BATD and DBATD. The increase in the inhibitor concentration was accompanied by a decrease in weight loss and corrosion rate and an increase in percentage inhibition efficiency (data not included in the text). These data lead to the conclusion that ATD, BATD and DBATD act

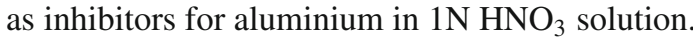




\subsection{Potentiodynamic polarization measurements}

Figure 2 shows anodic and cathodic polarization curves of aluminium in $1 \mathrm{~N} \mathrm{HNO}_{3}$ solution, without and with inhibitor molecules at room temperature $\left(25 \pm 1^{\circ} \mathrm{C}\right)$ after immersion for $1 \mathrm{~h}$. It is observed that both cathodic and anodic curves show lower current density in presence of the inhibitor molecules. This indicates that triazole precursors inhibit the corrosion process. The polarization measurements also clearly illustrate the fact that the inhibitor molecules under the studied conditions, brings down the corrosion current without causing any considerable change in the corrosion mechanism, suggesting that the inhibitors show mixed type behaviour. According to corrosion theory (Jones 1983), the rightward shift of the cathodic curves reveal that corrosion is mainly accelerated by cathode reactions. Additionally, the potentiodynamic polarization curves in figure 2 exhibit no steep slope in the anodic range, meaning that no passive films are formed on the aluminium surface. The shape of the graphs remain the same for these three different inhibitors and therefore, only the representative graphs are given in the text. Consequently, aluminium may directly dissolve in $1 \mathrm{~N} \mathrm{HNO}_{3}$ solutions. The electrochemical reactions for aluminium in $\mathrm{HNO}_{3}$ solution (McCafferty 2005; Khaled et al 2009; Zhang and Hua 2010) may be described as follows

$$
\begin{aligned}
& \mathrm{Al}_{(\mathrm{s})}+\mathrm{H}_{2} \mathrm{O} \leftrightharpoons \mathrm{AlOH}_{\mathrm{ads}}+\mathrm{H}^{+}+e \\
& \mathrm{AlOH}_{\mathrm{ads}}+\mathrm{H}_{2} \mathrm{O}+\mathrm{H}^{+} \leftrightharpoons \mathrm{Al}^{3+}+6 \mathrm{H}_{2} \mathrm{O}+2 e \\
& \mathrm{Al}^{3+}+\mathrm{H}_{2} \mathrm{O} \leftrightharpoons[\mathrm{AlOH}]^{2+}+\mathrm{H}^{+}, \\
& {[\mathrm{AlOH}]^{2+}+\mathrm{X}^{-} \leftrightharpoons[\mathrm{AlOHX}]^{+}}
\end{aligned}
$$

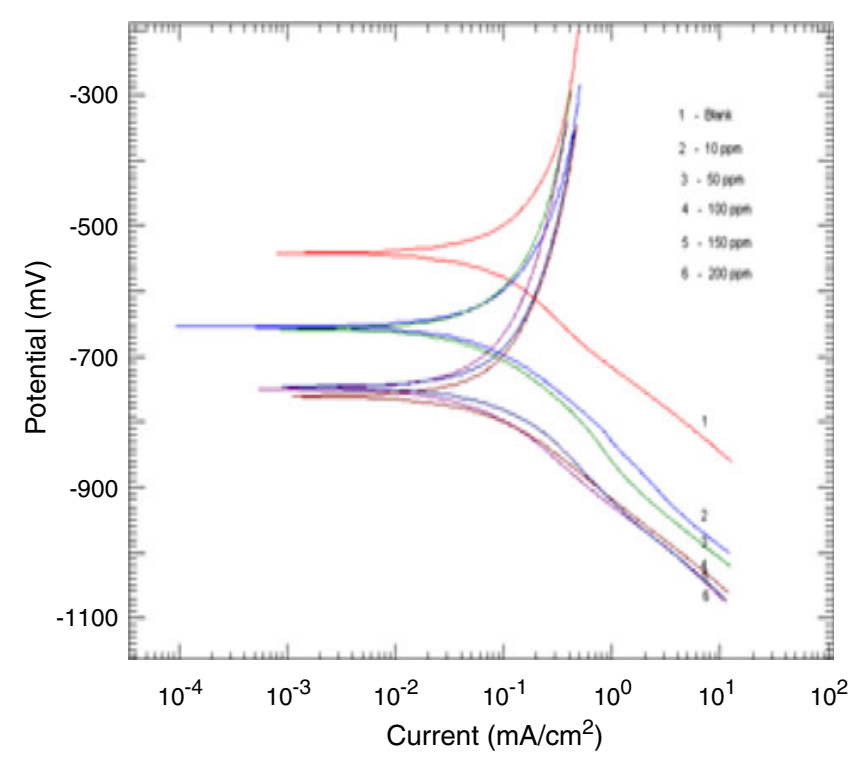

Figure 2. Anodic and cathodic tafel lines for aluminium in $1 \mathrm{~N}$ $\mathrm{HNO}_{3}$ with different concentrations of ATD inhibitors.
The controlling step in the metal dissolution is the complexation reaction between hydrated cation and anion $\mathrm{X}^{-}$(R4).

Figure 2 shows that with increasing concentrations of inhibitor molecules, the corrosion current density $\left(i_{\text {corr }}\right)$ decreased considerably. The maximum decrease in the corrosion current density was observed for DBATD. Inspection of polarization curves in figure 2 shows that it was not possible to evaluate the cathodic Tafel slope as there is no visible linear region that prevents linear extrapolation to $E_{\text {corr }}$ of the cathodic polarization curves. It has been shown that in the Tafel extrapolation method, use of both the anodic and cathodic Tafel regions is undoubtedly preferred over the use of only one Tafel region (Quartarone et al 2008). However, the corrosion rate can also be determined by Tafel extrapolation of either the cathodic or anodic polarization curve alone. If only one polarization curve is used, it is generally the cathodic curve which usually produces a longer and better defined Tafel region. Anodic polarization may sometimes produce concentration effects, due to passivation and dissolution, as noted above, as well as roughening of the surface which can lead to deviations from Tafel behaviour. The situation is quite different here; the anodic dissolution of aluminium in aerated $1 \mathrm{~N} \mathrm{HNO}_{3}$ solution obeys Tafel's law. The anodic curve is, therefore, preferred over the cathodic one for the Tafel extrapolation method. However, the cathodic polarization curve displays a limiting diffusion current due to the reduction of dissolved oxygen. Thus, the cathodic process is controlled by concentration polarization rather than activation polarization, which prevented linear extrapolation of the cathodic curves. Similar results were reported earlier also (Chetouani et al 2005; Amin and Khaled 2010; Labjar et al 2010). The values of electrochemical kinetic parameter, corrosion potential ( $\left.E_{\text {corr }}\right)$, corrosion current density $\left(i_{\text {corr }}\right)$ and Tafel slopes $\left(\beta_{\mathrm{a}}\right.$ and $\left.\beta_{\mathrm{c}}\right)$ are determined from these experiments by extrapolation method and listed in table 1 . The corrosion inhibition efficiency was calculated using the relation

$$
\% \mathrm{IE}=\frac{I_{\text {corr* }}-I_{\text {corr }}}{I_{\text {corr }}} \times 100,
$$

where $I_{\text {corr* }}$ and $I_{\text {corr }}$ are uninhibited and inhibited corrosion current densities, respectively determined by extrapolation of Tafel lines in the corrosion potential. The inhibitor molecules first adsorb on the metal surface by blocking the available reaction sites (Amin et al 2007). The surface coverage increases with the inhibitor concentration. The presence of defects on the metal surface permits free access to $\mathrm{H}^{+}$ions (Ashassi-Sorkhabi et al 2006) and a significant dissolution of metal takes place, followed by desorption of the surface film from the metal surface (Tsuru et al 1978). The formation of inhibitor film on the metal surface provides considerable protection to aluminium in acid environment. This film reduces the active surface area exposed to the corrosive medium and delays the hydrogen evolution and metal dissolution. 
Table 1. Polarization parameter and inhibition efficiency for corrosion of aluminium in $1 \mathrm{~N} \mathrm{HNO}_{3}$ solutions in absence and presence of different concentrations of inhibitors at room temperature.

\begin{tabular}{lccccccc}
\hline Inhibitor type & Conc. $(\mathrm{ppm})$ & $E_{\text {corr }}(\mathrm{mV}(\mathrm{SCE}))$ & $\beta_{\mathrm{a}}\left(\mathrm{mV} \mathrm{dec}^{-1}\right)$ & $\beta_{\mathrm{C}}\left(\mathrm{mV} \mathrm{dec}^{-1}\right)$ & $i_{\text {corr }}\left(\mu \mathrm{A} \mathrm{cm}^{-2}\right)$ & $\eta(\%)$ & C.R. (mm/yr) \\
\hline Blank & & -632 & 347 & 167 & $0 \cdot 1314$ & - & $1 \cdot 4304$ \\
& 10 & -601 & 287 & 167 & $0 \cdot 1101$ & $16 \cdot 21$ & $1 \cdot 1209$ \\
& 50 & -542 & 245 & 166 & $0 \cdot 0794$ & $39 \cdot 57$ & $0 \cdot 8650$ \\
ATD & 100 & -760 & 227 & 137 & $0 \cdot 0603$ & $54 \cdot 11$ & $0 \cdot 6568$ \\
& 150 & -748 & 198 & 113 & $0 \cdot 0526$ & $59 \cdot 96$ & $0 \cdot 5731$ \\
& 200 & -756 & 160 & 99 & $0 \cdot 0500$ & $61 \cdot 94$ & $0 \cdot 5456$ \\
& 10 & -675 & 283 & 164 & $0 \cdot 0797$ & $39 \cdot 34$ & $0 \cdot 8682$ \\
BATD & 50 & -741 & 273 & 144 & $0 \cdot 0732$ & $44 \cdot 29$ & $0 \cdot 7984$ \\
& 100 & -737 & 196 & 136 & $0 \cdot 0484$ & $63 \cdot 16$ & $0 \cdot 5278$ \\
& 150 & -648 & 99 & 77 & $0 \cdot 0465$ & $64 \cdot 61$ & $0 \cdot 5071$ \\
& 200 & -743 & 91 & 63 & $0 \cdot 0249$ & $81 \cdot 05$ & $0 \cdot 2717$ \\
& 10 & -681 & 281 & 167 & $0 \cdot 0761$ & $42 \cdot 05$ & $0 \cdot 8524$ \\
DBATD & 50 & -700 & 270 & 151 & $0 \cdot 0714$ & $45 \cdot 66$ & $0 \cdot 7714$ \\
& 100 & -717 & 185 & 144 & $0 \cdot 0471$ & $64 \cdot 23$ & $0 \cdot 5173$ \\
& 150 & -691 & 136 & 109 & $0 \cdot 0437$ & $66 \cdot 74$ & $0 \cdot 4870$ \\
& 200 & -692 & 103 & 99 & $0 \cdot 0214$ & $83 \cdot 17$ & $0 \cdot 2616$ \\
\hline
\end{tabular}

\subsection{Electrochemical impedance spectroscopy $($ EIS $)$}

Nyquist and Bode plots of ATD, BATD and DBATD in 1N $\mathrm{HNO}_{3}$ solution in the absence and presence of various concentrations of triazole derivatives are given in figures 3 and 4 . The shape of the graphs (Nyquist and Bode) remain the same for these three different inhibitors and therefore, only the representative graphs are given in the text. The impedance spectra show a single semicircle and the diameter of the semicircle increases with increasing inhibitor concentrations. These diagrams indicate that the impedance spectra consist of one capacitive loop at high frequency and the high frequency capacitive loop was attributed to charge transfer of the corrosion process (Bentiss et al 2000). Various parameters such as charge-transfer resistance $\left(R_{\mathrm{ct}}\right)$, double layer capacitance $\left(C_{\mathrm{dl}}\right)$ and $i_{\text {corr }}$ were obtained from impedance measurements and the results are given in table $2 . R_{\mathrm{ct}}$ values were calculated from the difference in impedance at lower and higher frequencies as suggested by Tsuru et al (1978). $C_{\mathrm{dl}}$ values were calculated from the frequency at which the imaginary component of impedance was maximum $\left(Z_{\max }\right)$ using the reaction

$$
C_{\mathrm{dl}}=\frac{1}{2 \pi f_{\max }} \frac{1}{R_{\mathrm{ct}}},
$$

where $f_{\max }$ is the frequency at which the imaginary component of impedance is maximum. The inhibition efficiency got from the charge-transfer resistance is calculated by the following relation:

$$
I E \%=\frac{R_{\mathrm{ct}}-R_{\mathrm{ct}}^{*}}{R_{\mathrm{ct}}} \times 100
$$

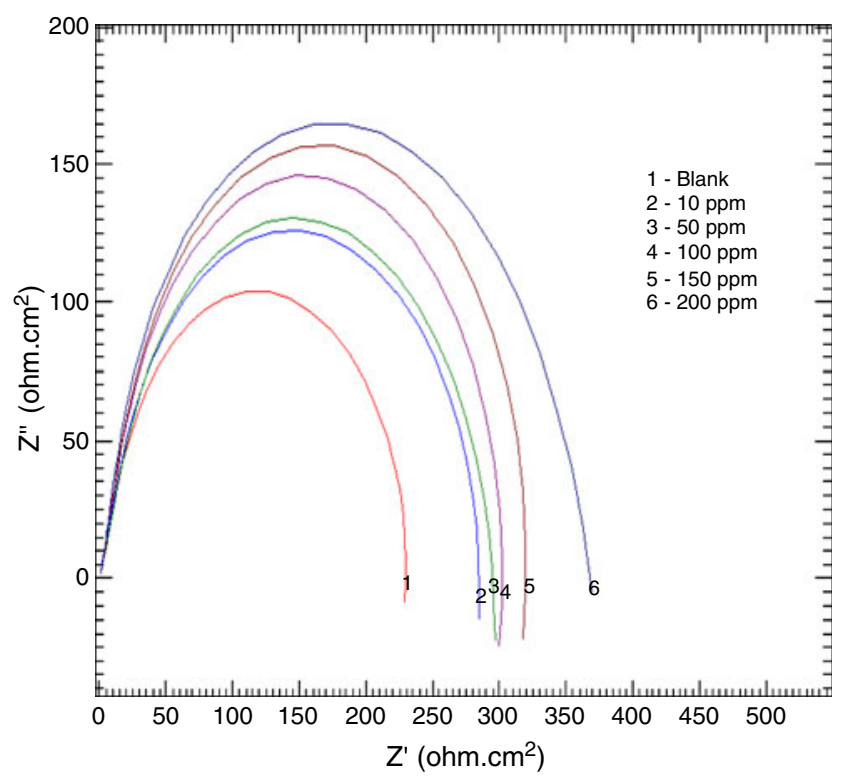

Figure 3. Nyquist diagrams for aluminium in $1 \mathrm{~N} \mathrm{HNO}_{3}$ containing different concentrations of ATD.

where $R_{\mathrm{ct}}$ and $R_{\mathrm{ct}}^{*}$ represent the resistance of charge transfer in the presence and absence of inhibitors, respectively. $R_{\mathrm{ct}}$ corresponds to the diameter of the loop and the $R_{\mathrm{ct}}$ values of ATD, BATD and DBATD increases with increasing inhibitor concentration whereas $C_{\mathrm{dl}}$ values show opposite trend. These observations support the fact that the corrosion of aluminium in $1 \mathrm{~N} \mathrm{HNO}_{3}$ is controlled by a charge transfer process. The decrease in $C_{\mathrm{dl}}$ is due to the gradual replacement of water molecules by the adsorption of the organic molecules at metal/solution interface, leading to a protective film on the metal (Wang et al 2007). 


\subsection{Quantum chemical calculations}

The energy of the highest occupied molecular orbital (HOMO), is a measure of electron donating ability of a molecule and explains the adsorption characteristics on metallic surface. A high $E_{\mathrm{HOMO}}$ value expresses intrinsic electron donating tendency to an appropriate acceptor, i.e. any molecule with lower HOMO energy and empty molecular orbital, while, $E_{\mathrm{LUMO}}$, the energy of the lowest unoccupied molecular orbital signifies the electron receiving

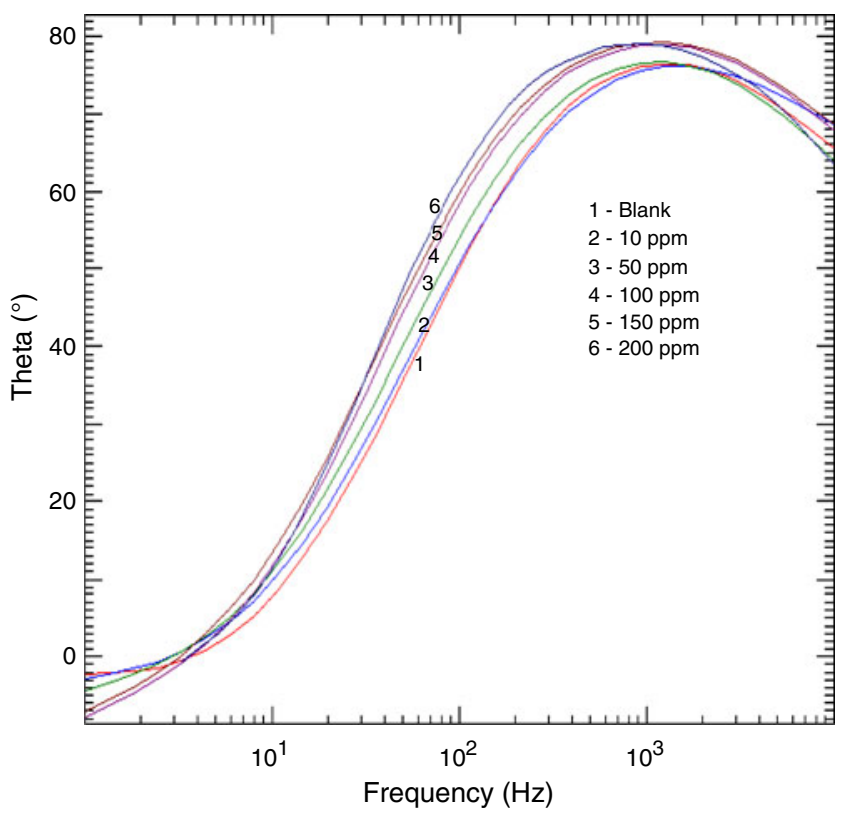

Figure 4. Bode diagrams for aluminium in $1 \mathrm{~N} \mathrm{HNO}_{3}$ containing different concentrations of ATD. tendency of a molecule. Accordingly, the difference between LUMO and HOMO energy levels, the dipole moment $(\mu)$, the electron charge on hetero-atoms and the total energy of inhibitor molecules were determined. Based on these, the calculated difference $\left(E_{\mathrm{LUMO}}-E_{\mathrm{HOMO}}\right)$ demonstrates inherent electron donating ability and measures the interaction of the inhibitor molecule with the aluminium surface. All these computational calculations have been performed in the gas phase. The highest occupied molecular orbital (HOMO) and the lowest unoccupied molecular orbitals (LUMO) are shown in figures 5 and 6 . According to the frontier molecular orbital theory of chemical reactivity, transition of electron is due to an interaction between the highest occupied molecular orbital and lowest unoccupied molecular orbital (LUMO) of reacting species. The energy of HOMO is directly related to the ionization potential and characterizes the susceptibility of the molecule toward attack by electrophiles. The energy of LUMO is directly related to the electron affinity and characterizes the susceptibility of the molecule toward attack by nucleophiles. The higher value of $E_{\mathrm{HOMO}}$ and lower value of the gap energy, $\Delta E$, show that DBATD acts as a better inhibitor than BATD and ATD. The calculated values of the ionization potential $(I)$ and the electron affinity $(A)$ by the application of Koopmans' theorem are shown in table 3. According to the Hartree-Fock theorem, a relationship exists between the energies of HOMO, LUMO, ionization potential and electron affinity as $-E_{\mathrm{HOMO}}=I ;-E_{\mathrm{LUMO}}=A$. Although no formal proof of this theorem exists within DFT, its validity is generally accepted. For $\chi$ and $\eta$, their operational and approximate definitions are

$$
-\mu=(I+A) / 2=\chi, \eta=(I-A) / 2 .
$$

Two systems, $\mathrm{Al}$ and inhibitor, are brought together, electrons will flow from lower $\chi$ (inhibitor) to higher $\chi(\mathrm{Al})$,

Table 2. Impedance parameter and inhibition efficiency for corrosion of aluminium in $1 \mathrm{~N} \mathrm{HNO}_{3}$ solutions in absence and presence of different concentrations of inhibitors at room temperature.

\begin{tabular}{|c|c|c|c|c|c|c|c|}
\hline Inhibitor & Conc. (ppm) & $R_{\mathrm{ct}}\left(\Omega \mathrm{cm}^{2}\right)$ & $C_{\mathrm{dl}}\left(\mu \mathrm{F} \mathrm{cm}^{-2}\right)$ & $i_{\text {corr }}\left(\mu \mathrm{A} \mathrm{cm}^{2}\right)$ & C.R. (mm/yr) & $n$ & $\eta(\%)$ \\
\hline \multirow[t]{3}{*}{ Blank } & & 225 & $12 \cdot 61$ & $0 \cdot 1158$ & $1 \cdot 262$ & $0 \cdot 96$ & - \\
\hline & 10 & 281 & $12 \cdot 41$ & 0.0926 & 1.0088 & 0.94 & $20 \cdot 06$ \\
\hline & 50 & 293 & $12 \cdot 32$ & $0 \cdot 089$ & 0.9696 & 0.94 & $23 \cdot 2$ \\
\hline \multirow[t]{5}{*}{ ATD } & 100 & 299 & $10 \cdot 89$ & 0.0872 & 0.9501 & 0.98 & $24 \cdot 72$ \\
\hline & 150 & 316 & $10 \cdot 7$ & 0.0825 & 0.8987 & 0.97 & $28 \cdot 8$ \\
\hline & 200 & 369 & $10 \cdot 69$ & 0.0757 & 0.7707 & 0.92 & 38.93 \\
\hline & 10 & 286 & $12 \cdot 36$ & $0 \cdot 086$ & 0.9981 & 0.95 & $21 \cdot 26$ \\
\hline & 50 & 297 & $11 \cdot 22$ & 0.0814 & 0.9491 & 0.96 & $24 \cdot 25$ \\
\hline \multirow[t]{5}{*}{ BATD } & 100 & 307 & $10 \cdot 67$ & 0.0772 & 0.9272 & 0.96 & $26 \cdot 54$ \\
\hline & 150 & 339 & $10 \cdot 54$ & 0.0735 & 0.8767 & 0.97 & $33 \cdot 66$ \\
\hline & 200 & 382 & $10 \cdot 31$ & 0.0718 & 0.7522 & 0.93 & 41.08 \\
\hline & 10 & 286 & $12 \cdot 1$ & 0.0817 & 0.9765 & 0.97 & $21 \cdot 21$ \\
\hline & 50 & 299 & $11 \cdot 05$ & 0.0794 & 0.9507 & 0.97 & $24 \cdot 74$ \\
\hline \multirow[t]{3}{*}{ DBATD } & 100 & 316 & $10 \cdot 17$ & 0.0744 & 0.9171 & 0.98 & $28 \cdot 83$ \\
\hline & 150 & 342 & $9 \cdot 87$ & 0.0728 & 0.8653 & 0.95 & $34 \cdot 12$ \\
\hline & 200 & 395 & $9 \cdot 56$ & 0.0702 & 0.7187 & 0.94 & 43.04 \\
\hline
\end{tabular}


until the chemical potentials become equal. As a first approximation, the fraction of electrons transferred (Sastri and Perumareddi 1997), $\Delta N$, will be given by

$$
\Delta N=\frac{\chi_{\mathrm{Al}}-\chi_{\mathrm{inhi}}}{2\left(\eta_{\mathrm{Al}}+\eta_{\mathrm{inhi}}\right)},
$$

where $\mathrm{Al}$ is the Lewis acid according to HSAB concept (Pearson 1963, 1998). The difference in electronegativity drives the electron transfer, and the sum of hardness parameters acts as resistance (Do 1980). In order to calculate the fraction of electrons transferred, a theoretical value for the electronegativity of bulk aluminium was used, $\chi_{\mathrm{Al}} \approx$ $3.23 \mathrm{eV}$, and a global hardness of $\eta_{\mathrm{Al}} \approx 0$, by assuming that for a metallic bulk $I=A$ because they are softer than the neutral metallic atoms. From table 3 , it is evident that the fraction of electrons transferred, $\Delta N$, is high for DBATD and hence it shows higher inhibition efficiency.

The local reactivity is analysed by means of the condensed Fukui function. The condensed Fukui functions allow us to distinguish each part of the molecule on the basis of its distinct chemical behaviour due to different functional groups

(a)

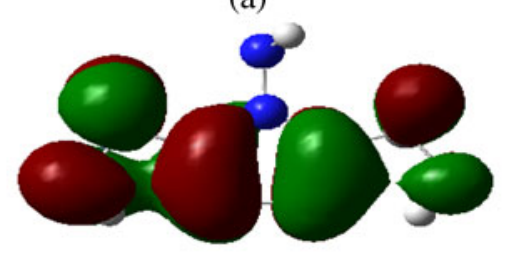

(b)

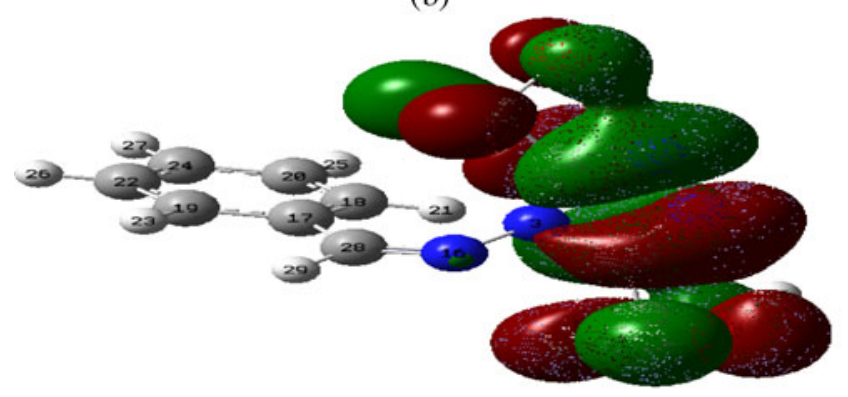

(c)

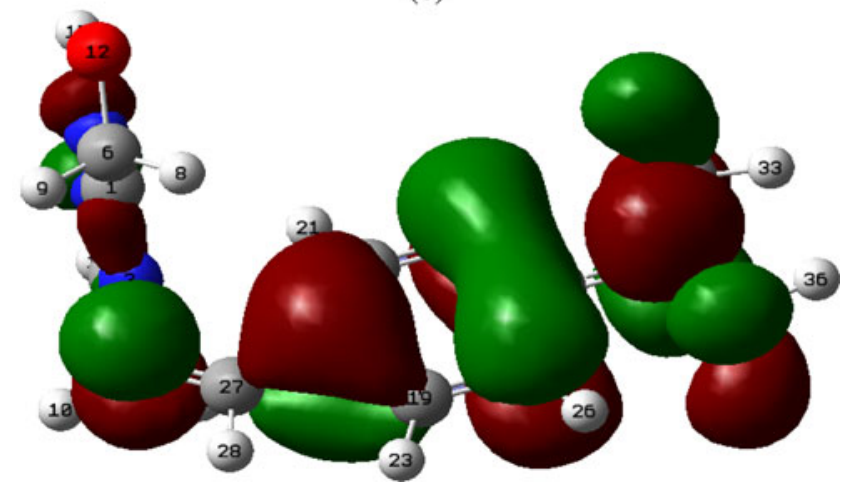

Figure 5. Highest occupied molecular orbital (HOMO) of inhibitors: (a) ATD, (b) BATD and (c) DBATD. or substituents. Thus, the site for nucleophilic attack will be the place where the value of $f_{k}^{+}$is a maximum and the site for electrophilic attack will be the place where the value of $f_{k}^{-}$is maximum. The values of the Fukui functions for a nucleophilic and electrophilic attack are given for the three inhibitors in table 4 (only for the nitrogen, oxygen and carbon atoms). Inspection of the values of Fukui functions presented in table 4 shows that DBATD has propitious zones for nucleophilic attack located on N16, C17, C20, C22, and N29 while BATD has only on C1, C2, N4 and N5. ATD has only nucleophilic centres on N3 and N4. Data in table 4 shows that DBATD has more susceptible sites for adsorption on the aluminium surface, which reflects its highest inhibition performance. The HOMO location on each system agrees with the atoms that exhibit greatest values of indices of Fukui, both indicate the zones by which the molecule would be adsorbed on the mild surface.

\subsection{Adsorption isotherm}

The nature of interaction between the inhibitor molecules and metal surface can be obtained from the adsorption isotherms.

(a)

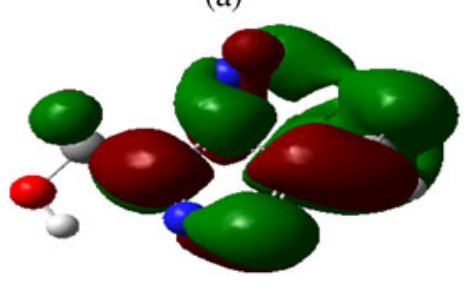

(b)

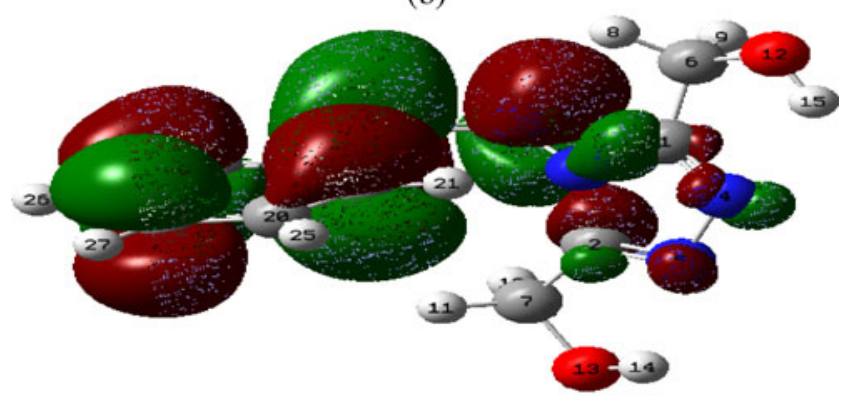

(c)

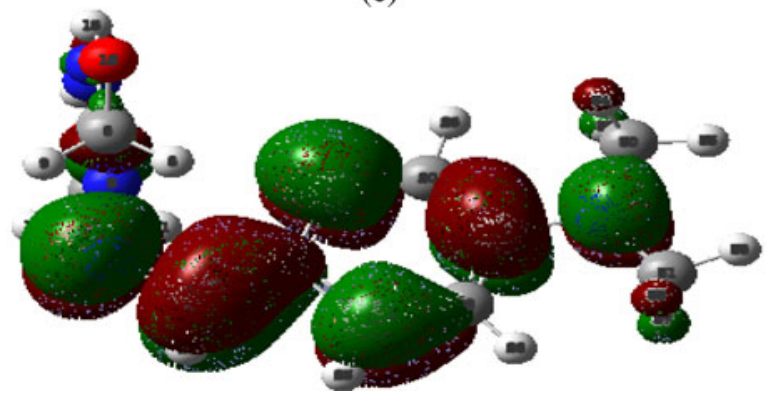

Figure 6. Lowest unoccupied molecular orbital (LUMO) of inhibitors: (a) ATD, (b) BATD and (c) DBATD. 
Table 3. Calculated quantum chemical properties for triazole derivatives ATD, BATD and DBATD.

\begin{tabular}{lccccccccccc}
\hline Molecule & Total energy $(\mathrm{eV})$ & HOMO $(\mathrm{eV})$ & LUMO $(\mathrm{eV})$ & $\Delta E(\mathrm{eV})$ & $\mu$ & $I$ & $A$ & $\chi$ & $\eta$ & $\Delta N$ \\
\hline ATD & -14330 & -0.1382 & -6.9805 & 6.8423 & 4.9367 & 0.1382 & 6.9805 & 3.5500 & -3.4212 & 0.0467 \\
BATD & -21653 & -2.4035 & -6.9190 & 4.5155 & 7.1341 & 2.4035 & 6.9190 & 4.6612 & -2.2577 & 0.3169 \\
DBATD & -25299 & -1.7910 & -5.9402 & 4.1491 & 9.5912 & 1.7910 & 5.9402 & 3.8656 & -2.0745 & 0.1532 \\
\hline
\end{tabular}

Table 4. Condensed Fukui functions for case when the molecule receives charge from metal surface.

\begin{tabular}{|c|c|c|c|c|c|c|c|c|}
\hline \multicolumn{3}{|c|}{ ATD } & \multicolumn{3}{|c|}{ BATD } & \multicolumn{3}{|c|}{ DBATD } \\
\hline Atoms & $f_{k}^{+}$ & $\overline{f_{k}^{-}}$ & Atoms & $f_{k}^{+}$ & $\overline{f_{k}^{-}}$ & Atoms & $f_{k}^{+}$ & $\overline{f_{k}^{-}}$ \\
\hline $\mathrm{C}(1)$ & 0.0268 & $0 \cdot 2664$ & $\mathrm{C}(1)$ & $0 \cdot 1639$ & 0.0103 & $\mathrm{C}(1)$ & 0.0068 & 0.0012 \\
\hline $\mathrm{C}(2)$ & 0.0265 & 0.2675 & $\mathrm{C}(2)$ & $0 \cdot 1596$ & 0.0113 & $\mathrm{C}(2)$ & 0.0061 & $0 \cdot 0012$ \\
\hline $\mathrm{N}(3)$ & $0 \cdot 2039$ & $0 \cdot 2025$ & $\mathrm{~N}(3)$ & 0.0035 & 0.0039 & $\mathrm{~N}(3)$ & 0.0043 & 0.0082 \\
\hline $\mathrm{N}(4)$ & 0.4807 & 0.0425 & $\mathrm{~N}(4)$ & $0 \cdot 1308$ & 0.0062 & $\mathrm{~N}(4)$ & 0.0160 & 0.0039 \\
\hline $\mathrm{N}(7)$ & $0 \cdot 1181$ & 0.0524 & $\mathrm{~N}(5)$ & 0.1529 & 0.0040 & $\mathrm{~N}(5)$ & 0.0176 & $0 \cdot 0043$ \\
\hline $\mathrm{N}(8)$ & $0 \cdot 1186$ & 0.0531 & $C(6)$ & 0.0302 & 0.0020 & $C(6)$ & 0.0009 & 0.0011 \\
\hline $\mathrm{C}(9)$ & 0.0009 & $0 \cdot 0122$ & $\mathrm{C}(7)$ & 0.0218 & 0.0011 & $\mathrm{C}(7)$ & 0.0007 & 0.0018 \\
\hline $\mathrm{O}(12)$ & 0.0533 & 0.0006 & $\mathrm{O}(12)$ & $0 \cdot 1166$ & 0 & $\mathrm{O}(12)$ & 0.0012 & $0 \cdot 0004$ \\
\hline$C(14)$ & 0.0009 & 0.0123 & $\mathrm{O}(13)$ & 0.0662 & 0.0002 & $\mathrm{O}(13)$ & 0.0011 & 0.0002 \\
\hline \multirow[t]{11}{*}{$\mathrm{O}(17)$} & 0.0053 & 0.0006 & $\mathrm{~N}(16)$ & 0.0014 & $0 \cdot 2204$ & $\mathrm{~N}(16)$ & $0 \cdot 1685$ & $0 \cdot 3837$ \\
\hline & & & $\mathrm{C}(17)$ & 0.0001 & 0.0798 & $\mathrm{C}(17)$ & $0 \cdot 3106$ & 0.0895 \\
\hline & & & $\mathrm{C}(18)$ & 0.0001 & $0 \cdot 1160$ & $\mathrm{C}(18)$ & 0.0128 & $0 \cdot 2310$ \\
\hline & & & $\mathrm{C}(19)$ & 0.0001 & 0.0898 & $\mathrm{C}(19)$ & 0.0154 & $0 \cdot 1624$ \\
\hline & & & $\mathrm{C}(20)$ & 0 & 0.0091 & $\mathrm{C}(20)$ & 0.2054 & 0.0061 \\
\hline & & & $\mathrm{C}(22)$ & 0.0001 & 0.0226 & $\mathrm{C}(22)$ & 0.2202 & $0 \cdot 0115$ \\
\hline & & & $\mathrm{C}(24)$ & 0 & $0 \cdot 1630$ & $\mathrm{C}(24)$ & 0.0855 & $0 \cdot 2558$ \\
\hline & & & $\mathrm{C}(28)$ & 0.0003 & $0 \cdot 2564$ & $\mathrm{C}(27)$ & 0.0102 & 0.4791 \\
\hline & & & & & & $\mathrm{N}(29)$ & 0.5524 & 0.0920 \\
\hline & & & & & & $\mathrm{C}(30)$ & 0.0108 & 0.0025 \\
\hline & & & & & & $\mathrm{C}(31)$ & 0.0102 & 0.0025 \\
\hline
\end{tabular}

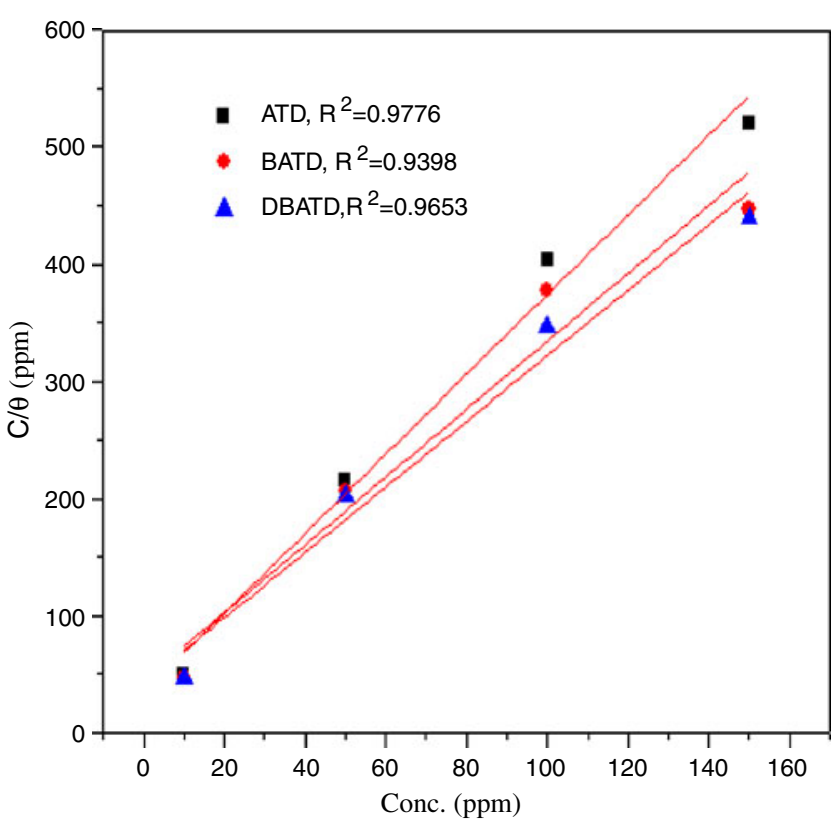

Figure 7. Langmuir's isotherm for adsorption of triazole derivatives in $1 \mathrm{M} \mathrm{HNO}_{3}$ on copper surface at $298 \mathrm{~K}$.
The values of surface coverage $(\theta)$, corresponding to different concentrations of inhibitors at $298 \mathrm{~K}$ have been used to get best linearity isotherm (Bouklah et al 2006). The adsorption isotherms generally considered were:

Temkin isotherm, $\exp f(\theta)=K_{\mathrm{ads}} C$,

Langmuir isotherm, $\frac{\theta}{1-\theta}=K_{\mathrm{ads}} C$,

Frumkin isotherm, $\frac{\theta}{1-\theta} \exp -2 f(\theta)=K_{\text {ads }} C$,

Freundlich isotherm, $\theta=K_{\mathrm{ads}} C$,

where $K_{\text {ads }}$ is the equilibrium constant of the inhibitor adsorption process, $C$ the inhibitor concentration and $f$ the factor of energetic in homogeneity, the values of surface coverage $(\theta)$ can be obtained from impedance measurements according to the equation

$$
\theta=\frac{R_{\mathrm{ct}}-R_{\mathrm{ct}}^{*}}{R_{\mathrm{ct}}} .
$$

The Langmuir isotherm was found to provide the best description of the adsorption behaviour. Plots of $C / \theta$ vs $C$ 
yield a straight line as shown in figure 7 . In both cases the linear regression coefficients $\left(R^{2}\right)$ are almost equal to 1 , indicating that the adsorption of ATD, BATD and DBATD obeys the Langmuir adsorption isotherm and $K_{\text {ads }}$ values can be calculated from the intercepts of the straight lines on the $C / \theta$-axis, the $K_{\text {ads }}$ is related to the standard free energy of adsorption, $\Delta G_{\text {ads }}^{0}$ with the following equation:

$$
\Delta G_{\mathrm{ads}}^{0}=-R T \ln \left(55 \cdot 5 K_{\mathrm{ads}}\right),
$$

the value 55.5 in the above equation is the molar concentration of water in solution in mol/l (John et al 2010a). Thermodynamic parameters for adsorption process obtained from Langmuir adsorption isotherm for the inhibitors are given in table 5. The negative value of $\Delta G_{\text {ads }}^{0}$ obtained on the addition of inhibitors indicate that adsorption of ATD, BATD and DBATD is spontaneous in nature (Flis and Zakroczymski 1996). It is generally accepted that the value of $\Delta G_{\text {ads }}^{0}$ up to $-20 \mathrm{~kJ} \mathrm{~mol}^{-1}$ indicates physisorption, the electrostatic interactions between charged molecules and charged metal, while the value around $-40 \mathrm{~kJ} \mathrm{~mol}^{-1}$ or smaller indicate typical chemisorptions, the charge sharing or charge transfer from the inhibitor molecules to the metal surface to form a covalent bond (Bentiss et al 2005; John et al 2010b). The values of $\Delta G_{\mathrm{ads}}^{0}$ in this measurement range from -22 to $-37 \mathrm{~kJ} \mathrm{~mol}^{-1}$ (table 5) which suggest that the adsorption of

Table 5. Equilibrium constant and standard free energy for aluminium in $1 \mathrm{~N} \mathrm{HNO}_{3}$ in presence of different concentrations of inhibitors.

\begin{tabular}{lrrc}
\hline Compound & Conc. & \multicolumn{1}{c}{$K_{\text {ads }}$} & $-\Delta G_{\text {ads }}\left(\mathrm{kJ} \mathrm{mol}^{-1}\right)$ \\
\hline \multirow{4}{*}{ ATD } & 10 & 2785 & $29 \cdot 8024$ \\
& 50 & 1885 & $29 \cdot 8292$ \\
& 100 & 169 & $22 \cdot 8244$ \\
& 150 & 1475 & $28 \cdot 2180$ \\
& 200 & 1171 & $27 \cdot 6424$ \\
BATD & 10 & 15046 & $34 \cdot 0091$ \\
& 50 & 3758 & $30 \cdot 5490$ \\
& 100 & 3977 & $30 \cdot 6906$ \\
& 150 & 2823 & $29 \cdot 8361$ \\
DBATD & 200 & 49614 & $36 \cdot 9850$ \\
& 10 & 19955 & $34 \cdot 7133$ \\
& 50 & 46216 & $36 \cdot 8081$ \\
& 100 & 4938 & $31 \cdot 2307$ \\
& 150 & 3678 & $30 \cdot 4959$ \\
& 200 & 67949 & $37 \cdot 6943$ \\
\hline
\end{tabular}

(a)

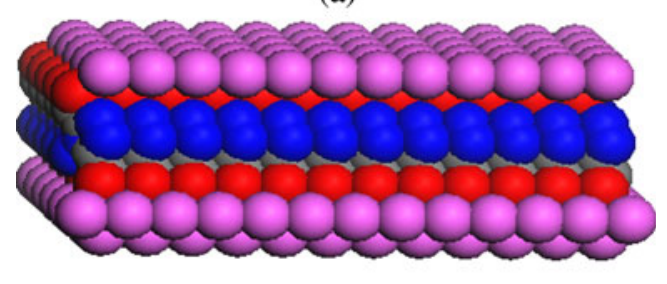

(b)

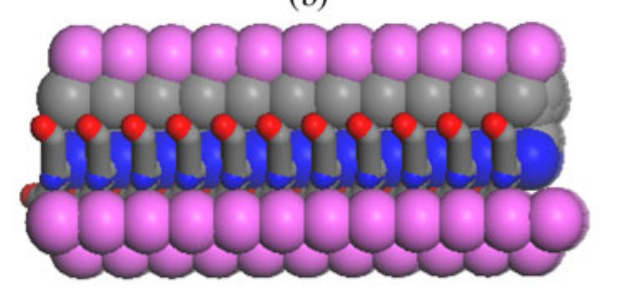

(c)

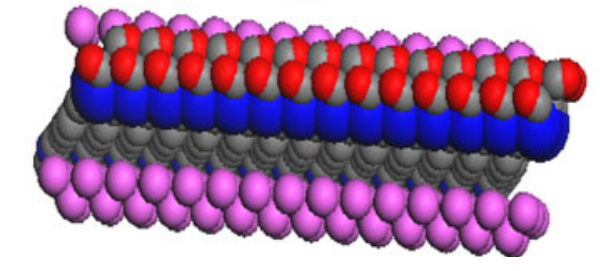

Figure 8. Modes of adsorption of (a) ATD, (b) BATD and (c) DBATD on $\mathrm{Al}$ (110) surface.

ATD, BATD and DBATD involves two types of interactions: chemisorption and physisorption.

\subsection{Molecular simulation study}

Molecular modeling studies provide a strong evidence for the molecular attraction of ATD, BATD and DBATD to the metal surface. The prediction of the inhibitor-surface interaction through the modeling studies lead to optimal molecular binding on the metal surface (Xia et al 2008; Khaled and Amin 2009).

In Monte Carlo simulation, molecular dynamics were performed on a system comprising inhibitors and aluminium (110) surface. Through Monte Carlo simulation process one can arrive at the conclusion that which out of the studied inhibitors have lowest energy. The outputs and descriptors calculated by Monte Carlo simulation are presented in table 6. The parameters presented in table 6 include total energy of the substrate-adsorbate configuration. The total

Table 6. Outputs and descriptors calculated by Monte Carlo simulation for adsorption of ATD, BATD and DBATD on Al (110) surface.

\begin{tabular}{lcccccc}
\hline Inhibitor & $\begin{array}{c}\text { Total energy } \\
\left(\mathrm{kJ} \mathrm{mol}^{-1}\right)\end{array}$ & $\begin{array}{c}\text { Adsorption energy } \\
\left(\mathrm{kJ} \mathrm{mol}^{-1}\right)\end{array}$ & $\begin{array}{c}\text { Rigid ad. energy } \\
\left(\mathrm{kJ} \mathrm{mol}^{-1}\right)\end{array}$ & $\begin{array}{c}\text { Deformation energy } \\
\left(\mathrm{kJ} \mathrm{mol}^{-1}\right)\end{array}$ & $\begin{array}{c}\text { Atomistic: } \\
\mathrm{d} E_{\mathrm{ad}} / \mathrm{d} N_{\mathrm{i}}\end{array}$ & $\begin{array}{c}\text { Calculated binding energy } \\
\left.(\mathrm{kJ} \mathrm{mol})^{-1}\right)\end{array}$ \\
\hline ATD & 10.32 & -45782.71 & 25.32 & -45808.04 & -45782.72 & 45511.59 \\
BATD & 704.26 & -63182.44 & 565.17 & -63786.97 & -63221.79 & 63390.72 \\
DBATD & 1518.72 & -73655.20 & 1003.85 & -74659.09 & -73655.24 & 73629.31 \\
\hline
\end{tabular}


(a)

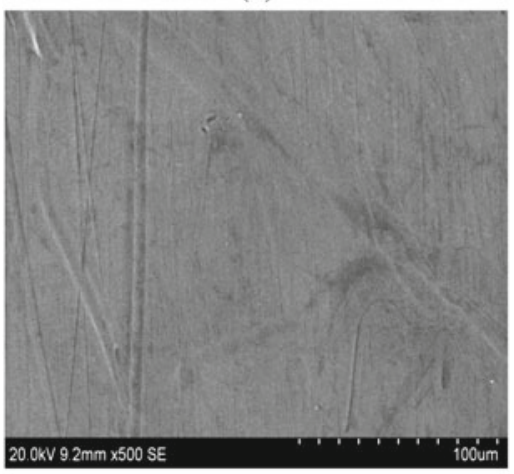

(c)

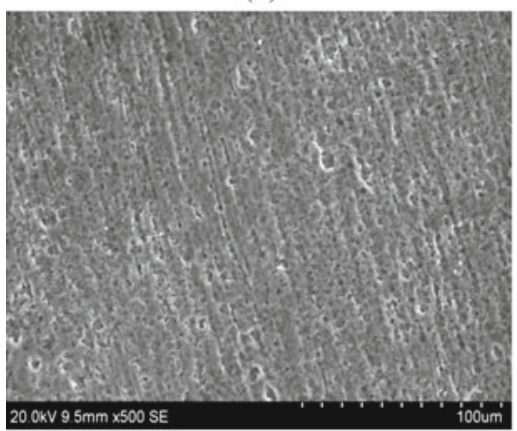

(b)

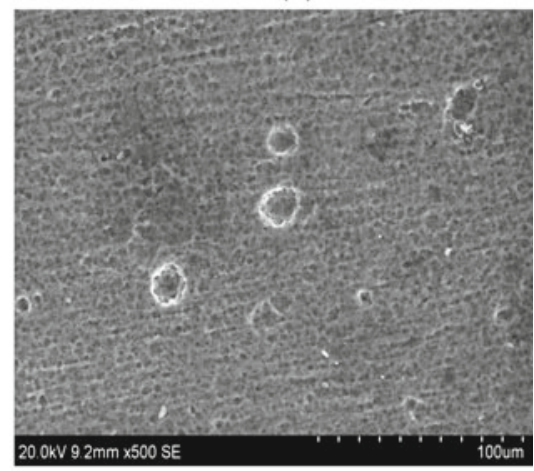

(d)

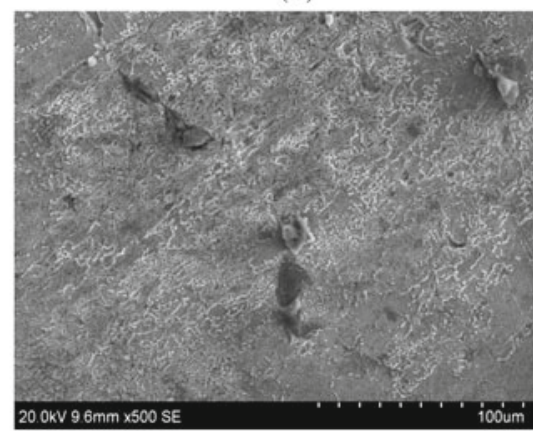

(e)

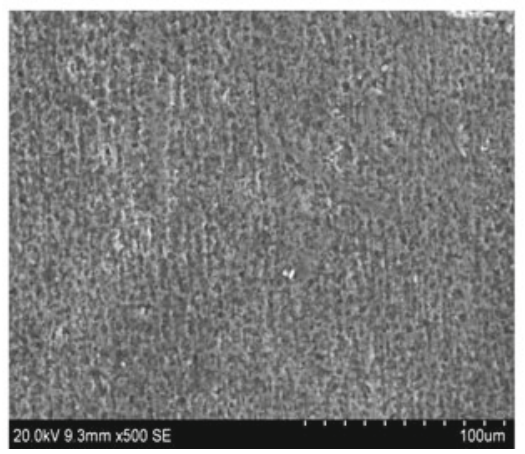

Figure 9. SEM images of (a) aluminium metal, (b) in $1 \mathrm{~N} \mathrm{HNO}_{3}$ without inhibitor, (c) in presence of $200 \mathrm{ppm}$ of ATD after $24 \mathrm{~h}$, (d) in presence of $200 \mathrm{ppm}$ of BATD after $24 \mathrm{~h}$ and (e) in presence of $200 \mathrm{ppm}$ of DBATD after $24 \mathrm{~h}$ weight loss measurements.

energy is defined as the sum of the energies of the adsorbate components, rigid adsorption energy and deformation energy. In this study, the substrate energy is taken as zero.

The adsorption energy is defined as the sum of the rigid adsorption energy and deformation energy for the adsorbate components. The rigid adsorption energy reports the energy, in $\mathrm{kcal} \mathrm{mol}^{-1}$, released (or required) when the unrelaxed adsorbate components are adsorbed on the substrate. The deformation energy reports the energy released when the adsorbed adsorbate components are relaxed on the substrate surface. $\mathrm{d} E_{\mathrm{a}} / \mathrm{d} N_{\mathrm{i}}$ which defines the energy of substrate-adsorbate configurations where one of the adsorbate components has been removed. As can be seen from table 6, DBATD has maximum total energy and rigid adsorption energy which is in conformity with the experimental results. The close contact between the inhibitor molecules and $\mathrm{Al}(110)$ surface as well as the best adsorption configuration for the studied compounds are shown in figure 8 .

\subsection{Scanning electron microscopy}

Surface examination using SEM was carried out to study the effect of inhibitors on the surface morphology of aluminium metal. Figure 9(a) shows SEM image of a polished aluminium sample. Figure 9(b) shows SEM image of 
aluminium surface after immersion in $1 \mathrm{~N} \mathrm{HNO}_{3}$ solution with no additives for $24 \mathrm{~h}$. This micrograph reveals that the surface was strongly damaged in the absence of inhibitor. Figures 9(c), (d) and (e) show SEM images of the surface of aluminium immersed for $24 \mathrm{~h}$ in $1 \mathrm{~N} \mathrm{HNO}_{3}$ solution containing $200 \mathrm{ppm}$ of the inhibitor molecules. The faceting observed in figures 9(c), (d) and (e) was gone and the surface was free from pits and it was smooth. It can be concluded that the rate of corrosion is less in the presence of inhibitors. Magnification of SEM images clearly shows that more polished surface is formed in the case of DBATD (Wang et al 2002; Babic-Samardzija et al 2005; Zhao et al 2005; Ramesh and Adhikari 2009). These observations also support the results of electrochemical studies and quantum chemical calculations pertaining to these inhibitors.

\section{Conclusions}

(I) The inhibitor molecules show good inhibitive efficiency for aluminium in aerated $1 \mathrm{~N} \mathrm{HNO}_{3}$.

(II) The percentage inhibition efficiency increases with increase in concentration of ATD, BATD and DBATD and decreases with longer exposure periods at $298 \mathrm{~K}$.

(III) Higher surface coverage on the metal surface was obtained in $1 \mathrm{~N} \mathrm{HNO}_{3}$ solution with higher inhibitor concentrations.

(IV) Results of polarization studies suggest that the inhibitors, ATD, BATD and DBATD, act as mixed type inhibitors.

(V) The inhibitor molecules adsorb on the metal surface and blocking the reaction sites. The surface area available for attack of the corrosive species decreases with increasing inhibitor concentrations.

(VI) Molecular dynamics simulations are performed to investigate the adsorption behaviour of ATD, BATD and DBATD on aluminium surface.

(VII) The relationship between efficiency of inhibition of aluminium corrosion in $1 \mathrm{~N} \mathrm{HNO}_{3}$ by ATD, BATD and DBATD and the $E_{\mathrm{HOMO}}, E_{\mathrm{LUMO}}, \mathrm{E}_{\mathrm{LUMO}}-E_{\mathrm{HOMO}}$ and $\Delta N$ were calculated by DFT method.

(VIII) The results of quantum chemical calculations and electroanalytical studies are in conformity with each other and the inhibitor molecule DBATD acts as better inhibitor than BATD and ATD.

\section{Acknowledgements}

The authors are grateful to the Kerala State Council for Science, Technology and Environment (KSCSTE) for financial support in the form of a major research project 018/SRSPS/2006/CSTE.

\section{References}

Abd El-Maksoud S A 2002 Corros. Sci. 44803

Agrawal R and Namboodhiri T K G 1990 Corros. Sci. 3037

Ailor W H 1971 Handbook of corrosion testing and evaluation (New York: John Wiley and Sons)

Ajmal M, Mideen A S and Quraishi M A 1994 Corros. Sci. 3679

Amin M A and Khaled K F 2010 Corros. Sci. 521194

Amin M A, Abed El-Rehim S S, El-Sherbini E E F and Bayyomi R S 2007 Electrochim. Acta $\mathbf{5 2} 3588$

Arslan T, Kandemirli F, Ebenso E E, Love I and Alemu H 2009 Corros. Sci. $\mathbf{5 1} 35$

Ashassi-Sorkhabi H, Ghalebsaz-Jeddi N, Hashemzadeh F and Jahani H 2006 Electrochim. Acta $\mathbf{5 1} 3848$

Babic-Samardzija K, Lupu C, Hackerman N and Barron A R 2005 J. Mater. Chem. 151908

Bag S K, Chakraborty S B and Chaudhari S R 1993 J. Indian Chem. Soc. 7024

Bentiss F, Traisnel M and Lagrene M 2000 Corros. Sci. 42 127

Bentiss F, Lebrini M and Lagrene M 2005 Corros. Sci. 472915

Bouklah M, Hammouti B, Lagrene M and Bentiss F 2006 Corros. Sci. 482831

Chetouani A, Hammouti B, Benhadda T and Daoudi M 2005 Appl. Surf. Sci. 249375

Dessai M N, Dessai M B, Shah C B and Dessai S M 1986 Corros. Sci. 26827

Do D 1980 Adsorption analysis in equilibria and kinetics (London: Imperial College Press)

Ebenso E E, Arslan T, Kandemirli F, Caner N and Love I 2010 Int. J. Quantum Chem. 1101003

Flis J and Zakroczymski T 1996 J. Electrochem. Soc. 1432458

Frisch M J et al 2003 Gaussian 03 (Pittsburgh: Gaussian Inc.)

Gece G 2008 Corros. Sci. 502981

John S, Joseph B, Aravindakshan K K and Joseph A 2010a Mater. Chem. Phys. 122374

John S, Joseph B, Balakrishnan K V, Aravindakshan K K and Joseph A 2010b Mater. Chem. Phys. 123218

Jones D A 1983 Principles and prevention of corrosion (NJ: Prentice Hall) 2nd ed.

Khaled K F and Al-Qahtani M M 2009 Mater. Chem. Phys. 113 150

Khaled K F and Amin M A 2009 Corros. Sci. 511964

Khaled K F, Fadl-Allah S A and Hammouti B 2009 Mater. Chem. Phys. 117148

Klingele M H, Moubaraki B, Murray K S and Brooker S 2005 Chem. Eur. J. 11692

Labjar N, Lebrini M, Bentiss F, Chihib N E, Hajjaji S E and Jama C 2010 Mater. Chem. Phys. 119330

McCafferty E 2005 Corros. Sci. 473202

McCafferty E, Pravadic V and Zettlemoyer A C 1999 Trans. Faraday Soc. 66237

Pearson R G 1963 J. Am. Chem. Soc. 853533

Pearson R G 1988 Inorg. Chem. 27734

Pourbaix M 1966 Atlas of electrochemical equilibria in aqueous solutions (New York: Pergamon Press)

Quartarone G, Battilana M, Bonaldo L and Tortato T 2008 Corros. Sci. 503467

Ramesh S V and Adhikari A V 2009 Mater. Chem. Phys. 115 618

Sastri V S and Perumareddi J R 1997 Corros. Sci. 53617 
Solmaz R, Kardas G, Culha M, Yazici B and Erbil M 2008 Electrochim. Acta 535941

Talati J D and Modi R M 1975 Br. Corros. J. 10103

Tsuru T, Haruyama S and Gijutsu B 1978 J. Jpn. Soc. Corros. Eng. 27573

Wang H L, Fan H B and Zheng J S 2002 Mater. Chem. Phys. 77655
Wang H, Wang X, Wang H, Wang L and Liu A 2007 J. Mol. Model 13147

Xia S, Qiu M, Yu L, Liu F and Zhao H 2008 Corros. Sci. 502021

Yang W and Mortier W J 1986 J. Am. Chem. Soc. 1085708

Zhang Q B and Hua Y X 2010 Mater. Chem. Phys. 11957

Zhao P, Liang Q and Li Y 2005 Appl. Surf. Sci. 2521596 